\title{
FEASIBILITY OF NEAR-FIELD ODR IMAGING OF MULTI-GEV ELECTRON BEAMS AT CEBAF*
}

\author{
A.H. Lumpkin, Argonne Accelerator Institute, Argonne National Laboratory, Argonne, IL U.S.A \\ P. Evtushenko**, A.P. Freyberger**, and C.Y. Liu**, Jefferson Lab, Newport News, VA U.S.A.
}

\section{Abstract}

We have evaluated the feasibility of using the optical diffraction radiation (ODR) generated as a $1-$ to $6-\mathrm{GeV}$ $\mathrm{CW}$ electron beam passes nearby the edge of a single metal conducting plane as a nonintercepting (NI) relative beam size monitor for CEBAF. Previous experiments were successfully done using near-field imaging on the lower-current, 7-GeV beam at APS, and an analytical model was developed for near-field imaging. Calculations from this model indicate sufficient beam size sensitivity in the ODR profiles for beam sizes in the 30- to 50micron regime as found in the transport lines of CEBAF before the experimental targets. With anticipated beam currents of $100 \mu \mathrm{A}$, the ODR signal from the charge integrated over the video field time should be $\sim 500$ times larger than in the APS case. These signal strengths will allow a series of experiments to be done on beam energy dependencies, impact parameters, polarization effects, and wavelength effects that should further elucidate the working regime of this technique and test the model. Plans for the diagnostics station that will provide reference optical transition radiation (OTR) images will also be described.

\section{INTRODUCTION}

The monitoring of the electron beams used for the various nuclear physics experiments in the three halls at the Continuous Electron Beam Accelerator Facility (CEBAF) is an ongoing interest due to some beam-size variations that have been observed. The present harp systems are intercepting to the beam and are generally used in an off-experiment mode. We have evaluated the feasibility of using optical diffraction radiation (ODR) monitoring as a nonintercepting (NI) means to track the relative beam size of the $1-$ to $6-\mathrm{GeV}$ beams with up to $100 \mu \mathrm{A}$ of average current as they pass by a single conducting plane. Previous experiments were successfully done using near-field imaging on the lower-current, 7$\mathrm{GeV}$ beam at APS, and an analytical model was developed for near-field imaging $[1,2]$. Calculations from this model indicate sufficient beam size sensitivity in the ODR profiles for beam sizes in the 30-50 micron regime as found in the transport lines of CEBAF before the experimental targets. With the higher beam currents of CEBAF, a series of experiments should be possible on

\footnotetext{
*Work supported by the U.S. Department of Energy, Office of Science, Office of Basic Energy Sciences, under Contract No. DE-AC02$06 \mathrm{CH} 11357$.

**Authored by Jefferson Science Associates, LLC under U.S. DOE Contract No. DE-AC05-06OR23177. The U.S. Government retains a non-exclusive, paid-up, irrevocable, world-wide license to publish or reproduce this manuscript for U.S. Government purposes.
}

beam energy dependencies, impact parameters, polarization effects, and wavelength effects that should further elucidate the working regime of this technique and test the model. Although we have found the orthogonal polarization component for a spatial dimension has the most direct sensitivity to beam-size and centroid effects experimentally, for some cases the horizontal polarization component also has a unique feature that is also beamsize dependent [3]. We expect to be able to test this latter feature at the CEBAF test station as well in the 50- to $100-\mu \mathrm{m}$ beam-size regime.

\section{BACKGROUND}

\section{CEBAF Complex}

The CEBAF uses a DC photocathode (PC) gun based on a GaAs photocathode, a $62-\mathrm{MeV}$ injector, and a recirculating superconducting $\mathrm{rf}$ (SRF) linear accelerator. The beam from the injector is introduced into the two $500-\mathrm{MeV}$ linacs on either side of the racetrack ellipse. A series of dipoles at the entrance to the arcs allows the successive passes to be at energies from 1-6 GeV with an average current of $100 \mu \mathrm{A}$ [4]. A schematic of the facility is shown in Fig. 1. There is a tune-up mode with a $250-\mu \mathrm{s}$ macropulse with a $499-\mathrm{MHz}$ micropulse repetition rate. Extraction from the arcs brings the beam into the transport lines to the nuclear physics experiments in Halls A, B, and C. A beam kicker allows the distribution of the desired beam intensity to the selected halls. The arcs and facility have been designed to accommodate higher beam energies, and a 12-GeV upgrade has been proposed [5].

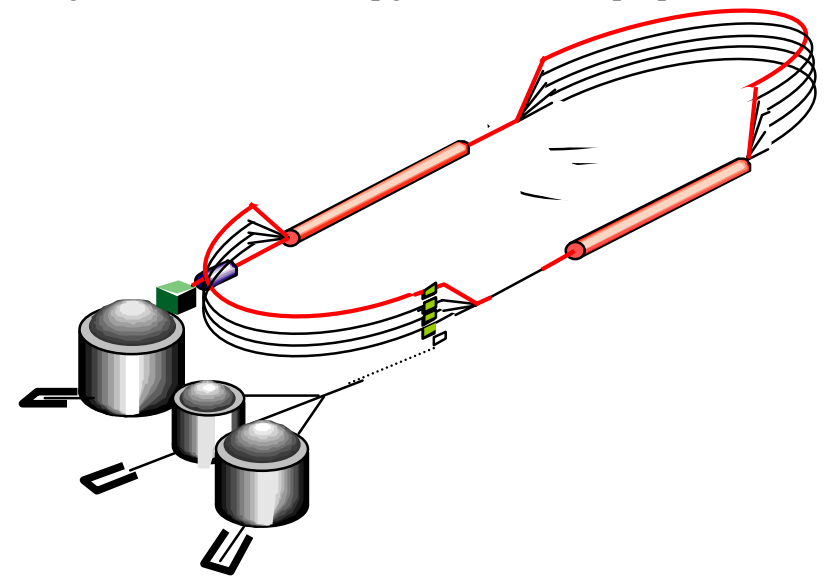

Figure 1: Schematic of the CEBAF recirculating SRF linac and the experimental halls. Beam can nominally be extracted at $1,2,3,4$, and $5 \mathrm{GeV}$ from the respective passes, and more recently the top energy is $6 \mathrm{GeV}$ via increased accelerator gradients. 


\section{ODR Background}

We propose to use the same techniques as demonstrated at APS where the optical transition radiation (OTR) images of the $7-\mathrm{GeV}$ beam were defined as the reference beam sizes for the ODR tests. By using the same imaging optics, magnifications, and camera for both types of data, we have a direct comparison of the ODR images to OTR images. For completeness we simply present that ODR is generated when a charged particle beam passed near a vertically inserted metal plane. In our simplified configuration we only have a single metal plane or screen, and we use optical lenses to integrate over the emission angles and frequency while focused at the screen or nearfield image. Based on the method of virtual quanta described by Jackson [6], we address the photon-like fields of relativistic beams. We convolve the electron beam's Gaussian distribution of sizes $\sigma_{x}$ and $\sigma_{y}$ with the field expected from a single electron at point $P$ in the metal plane. We wish to calculate the incoherent sum of radiation from all beam particles in a pulse emitted from a given point on the ODR radiator, i.e., at $\boldsymbol{u}=\boldsymbol{P}-\boldsymbol{r}_{\mathrm{o}}$, where $\boldsymbol{P}$ is the field point with respect to the origin and $\boldsymbol{r}_{\mathrm{o}}$ is the position of the beam centroid with respect to the origin. The impact parameter is $\boldsymbol{b}=\boldsymbol{u}-\boldsymbol{r}$, where $\boldsymbol{r}=\boldsymbol{r}(x, y)$ denotes a position in the beam measured from the beam centroid. We then can write the differential spectral intensity as:

$$
\begin{gathered}
\frac{d I}{d \omega}(\mathbf{u}, \omega)=\frac{1}{\pi^{2}} \frac{q^{2}}{c}\left(\frac{c}{\mathrm{v}}\right)^{2} \alpha^{2} N \frac{1}{\sqrt{2 \pi \sigma_{x}{ }^{2}}} \frac{1}{\sqrt{2 \pi \sigma_{y}{ }^{2}}} \times \\
\iint d x d y K_{1}{ }^{2}(\alpha b) e^{-\frac{x^{2}}{2 \sigma_{x}^{2}}} e^{-\frac{y^{2}}{2 \sigma_{y}{ }^{2}}}
\end{gathered}
$$

where $\omega=$ radiation frequency, $\mathrm{v}=$ particle velocity, $c=$ speed of light, $q$ = electron charge, $N$ is the particle number, $\alpha=1 / \gamma \lambda$, and $K_{1}(\alpha b)={ }_{K_{1}}\left(\alpha \sqrt{\left(u_{x}-x\right)^{2}+\left(u_{y}-y\right)^{2}}\right)$ is a modified Bessel function. Since we measure light intensity $I$, this should be proportional to $\left|E_{x}\right|^{2}+\left|E_{y}\right|^{2}$, resulting in the $K_{1}^{2}$ dependence. The incoherent photon intensity is proportional to $N$, the number of electrons, in contrast to the case of coherent diffraction radiation in the far infrared (FIR) [7], which is enhanced by $N^{2}$. An example of the calculated ODR intensity distribution for a $\sigma_{\mathrm{x}}=1375 \mu \mathrm{m}$ beam and impact parameter starting at 1.25 $\mathrm{mm}$ is shown in Fig. 2. Such an image can be fitted to a Gaussian distribution in the horizontal dimension as well, and beam size sensitivity evaluated.

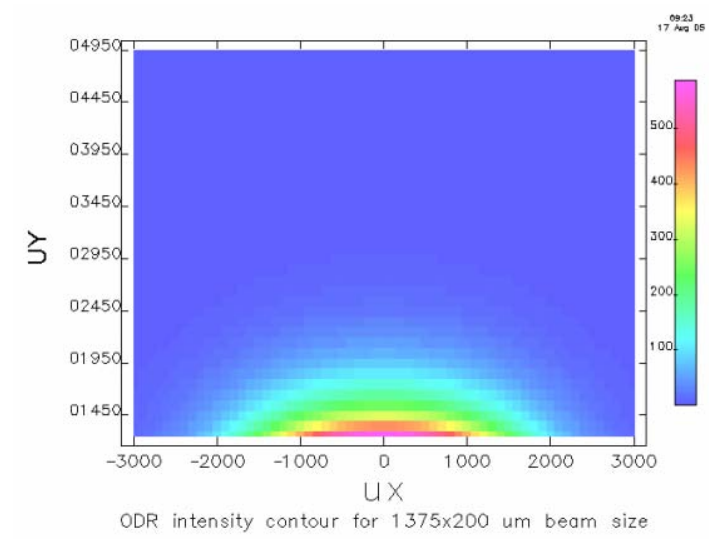

Figure 2: Calculation of the near-field ODR radiation from a single metal plane for a beam size of $1375 \mu \mathrm{m}$ (Ref. 2).

\section{ODR FEASIBILITY CONSIDERATIONS}

The extension of the APS style near-field ODR monitoring techniques should be a constructive exercise. The beam parameters of CEBAF are specifically of interest because the beam size is about 10-20 times smaller than the $1300-\mu \mathrm{m} \times$ transverse beam size at APS, and the average current is about 500 times more intense in a video field time of $16.6 \mathrm{~ms}$. The lattice values in a transport line to a target and the corresponding beam sizes as a function of the $\mathrm{z}$ location in the transport line were reviewed. Towards the target position we see a region of $50-\mu \mathrm{m}$ beam sizes. The comparison is summarized in Table 1. In addition, we note that the projected ILC linac beam sizes in the horizontal plane are comparable to the CEBAF values, so this is a very interesting regime to explore. More recently, an APS upgrade has been proposed based on an SRF energy recovering linac (ERL) operating at $7 \mathrm{GeV}$ with $100-\mathrm{mA}$ average current. Its projected beam sizes will be in the tens of microns also, so successful experiments at CEBAF will be a significant step forward in demonstrating feasibility for these future applications. The simple modeling results are reproduced from Ref. 2, and, as shown in Fig. 3, there is a clear change in the calculated ODR image profile for a beam size change from 20 to $50 \mu \mathrm{m}$. This is a total intensity calculation, so we would expect even more sensitivity to this change in the orthogonal polarization component [2].

Table 1: Summary of Some Relevant Parameters for APS, CEBAF, and ILC

\begin{tabular}{|l|c|c|c|c|}
\hline Parameter & APS & CEBAF & ILC & $\begin{array}{c}\text { APS } \\
\text { Upgrade }\end{array}$ \\
\hline Energy $(\mathrm{GeV})$ & 7 & 5 & $5-250$ & $1-7$ \\
\hline $\begin{array}{l}\text { x beam size } \\
(\mu \mathrm{m})\end{array}$ & 1300 & $30-50$ & 50,10 & $50-10$ \\
\hline $\begin{array}{l}\text { y beam size } \\
(\mu \mathrm{m})\end{array}$ & 200 & $30-50$ & 5,1 & $50-10$ \\
\hline $\begin{array}{l}\text { Current }(\mathrm{nA}) \\
\begin{array}{l}\text { Charge/16.6 } \\
\mathrm{ms}(\mathrm{nC})\end{array}\end{array}$ & 6 & 100,000 & $100,000,000$ & $1,000,000$ \\
\hline
\end{tabular}




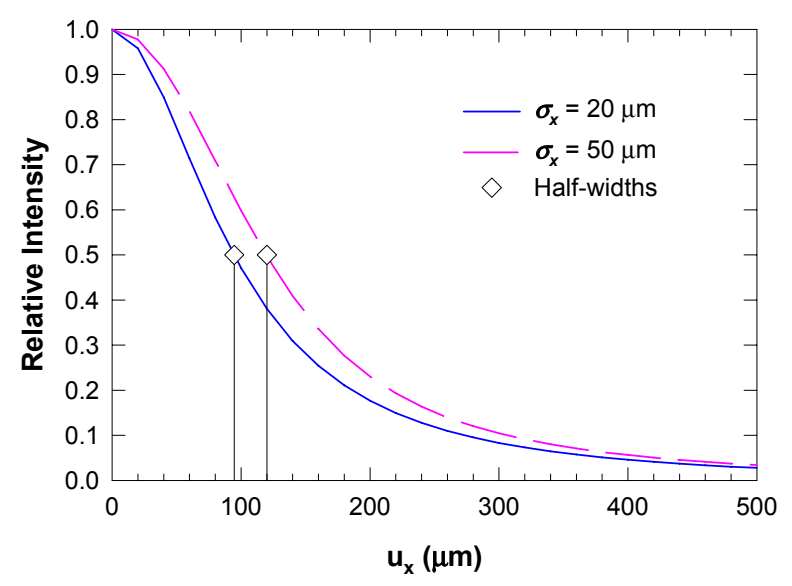

Figure 3: Analytical ODR results for the beam size sensitivity at the 20 - to $50-\mu \mathrm{m}$ regime. A clear change in the ODR profile size is seen between the two cases. Even more sensitivity should be seen with the orthogonal polarization component only (Ref. 2).

\section{OTR/ODR Station Design}

The diagnostics station (see Fig. 4) is designed with the flexibility of a stepper assembly to position an optical calibration scene, an OTR foil, or the ODR converter at selected impact-parameter values relative to the beam center. We propose to measure the reference beam sizes at low average current using OTR from the very thin, few$\mu \mathrm{m}$ metal foil oriented at 45 degrees to the beam direction and then to use the stepper assembly to explore ODR beam size sensitivity for impact parameter, beam intensity, beam energy, wavelength effects, and the two polarization components. Filter wheel assemblies will be used to select the appropriate neutral density filters, bandpass filters, and polarizers before the camera. As described previously [2], the ODR vertical polarization component more directly measures the horizontal beam size for the single plane metal surface inserted vertically near the beam path. However, there are also new modeling results [3] that show, for a given beam size and impact parameter, the horizontal polarization component of the near-field image can have a two-lobe feature whose central minimum visibility depends on beam size.

\section{SUMMARY}

In summary, the feasibility of applying the ODR nearfield, beam-size monitor technique to the beams before the CEBAF experimental halls looks very promising. Actual tests in one of the beamlines should help to



Figure 4: A CAD model drawing of the proposed OTR/ODR test station for the transport line showing the cube, optical transport, filter wheels, and camera locations. The alignment laser is shown at the left end of the girder.

identify any unexpected background sources or other operational issues. The next step would be to install several stations in the transport lines to provide the online feedback for beam size (emittance) for all three halls. We also anticipate that the scaling to $12-\mathrm{GeV}$ beams for the CEBAF upgrade will be straightforward if we perform the parameter scaling validations successfully from 1-6 GeV. In addition, the potential for applications to x-ray FELS, ERLs (APS Upgrade), and the ILC can be evaluated based on these tests at CEBAF.

\section{REFERENCES}

[1] Alex H. Lumpkin, NIMA 557, 318 (2006).

[2] A.H. Lumpkin et al., Phys. Rev. ST-AB 10, 022802 (2007), and references therein.

[3] C.Y. Yao, A.H. Lumpkin, and D.W. Rule, "Numerical Simulation of Optical Diffraction Radiation from a 7GeV Beam," these proceedings.

[4] C.R. Leeman, D.R. Douglas, and G.A. Kraft, Annu. Rev. Nucl. Part. Sci. 51, 413-50 (2001).

[5] Leigh Harwood, "Upgrading CEBAF to $12 \mathrm{GeV}$," Proceedings of PAC03, http://www.jacow.org.

[6] J.D. Jackson, Classical Electrodynamics (John Wiley and Sons, New York, 1975), Sec.15.4.

[7] A.H. Lumpkin, N.S. Sereno, and D.W. Rule, Nucl. Instrum. Methods A 475, 470 (2001). 